\title{
Loss of phosphatase and tensin homolog (PTEN) in myeloid cells controls inflammatory bone destruction by regulating the osteoclastogenic potential of myeloid cells
}

\author{
Stephan Blüml, ${ }_{1}^{1}$ Martin Friedrich, ${ }^{2}$ Tobias Lohmeyer, ${ }^{2}$ Emine Sahin, ${ }^{2}$ \\ Victoria Saferding, ${ }^{1}$ Julia Brunner, ${ }^{2}$ Antonia Puchner, ${ }^{1}$ Peter Mandl, ${ }^{1}$ \\ Birgit Niederreiter, ${ }^{1}$ Josef S Smolen, ${ }^{1}$ Gernot Schabbauer, ${ }^{2}$ Kurt Redlich $^{1}$
}

\begin{abstract}
Handling editor Tore K Kvien
- Additional material is published online only. To view please visit the journal online (http://dx.doi.org/10.1136/ annrheumdis-2013-203486).

${ }^{1}$ Division of Rheumatology, Internal Medicine III, Medical University of Vienna, Vienna, Austria

${ }^{2}$ Institute for Physiology, Center for Physiology and Pharmacology, Medical University Vienna, Vienna, Austria
\end{abstract}

\section{Correspondence to} Dr Kurt Redlich, Division of Rheumatology, Internal Medicine III, Medical University of Vienna, Währinger Gürtel 18-20, Vienna 1090, Austria; kurt.redlich@meduniwien.ac.at Gernot Schabbauer, Center for Biomolecular Medicine and Pharmacology, Medical University Vienna. A-1090 Vienna, Austria; gernot. schabbauer@meduniwien.ac.at

Received 18 February 2013 Revised 21 August 2013 Accepted 8 September 2013 Published Online First 27 September 2013

\section{CrossMark}

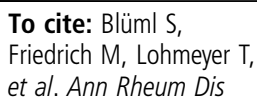

\section{ABSTRACT}

Objective Local bone destruction in rheumatic diseases, which often leads to disability and severely reduced quality of life, is almost exclusively mediated by osteoclasts. Therefore, it is important to understand pathways regulating the generation of osteoclasts. Here, we analysed the impact of the Phosphoinositide-3Kinase (PI3K)/Phosphatase and tensin homolog (PTEN) axis on osteoclast generation and bone biology under basal and inflammatory conditions.

Methods We analysed osteoclastogenesis of wildtype (wt) and PTEN ${ }^{-1-}$ cells in vitro and in vivo, pit resorption and $\mathrm{qPCR}$ of osteoclasts in vitro. Mice with a myeloid cell-specific deletion of PTEN and wt littermate mice were investigated by bone histomorphometry and clinical and histological assessment in the human tumour necrosis factor (TNF)-transgenic (hTNFtg) arthritis model. Results We show that myeloid-specific PTEN ${ }^{-/-}$mice display increased osteoclastogenesis in vitro and in vivo compared to wt mice. Loss of PTEN did not affect the generation or survival of osteoclast precursor cells. However, PTEN deficiency greatly enhanced receptor activator of nuclear factor $\kappa-B$ ligand (RANKL)-induced expression of the master transcription factor of osteoclastogenesis, nuclear factor of activated T-cells, cytoplasmic 1 (NFATC1), resulting in markedly increased terminal differentiation of osteoclasts in vitro. We also observed increased osteoclastogenesis under inflammatory conditions in the hTNFtg mouse model of arthritis, where hTNFtg/myeloid-specific PTEN ${ }^{-1-}$ mice displayed enhanced local bone destruction as well as osteoclast formation in the inflamed joints. The extent of synovial inflammation, however, as well as recruitment of osteoclast precursor cells was not different between wt and myeloid-specific PTEN ${ }^{-l-}$ mice.

Conclusions These data demonstrate that loss of PTEN and, therefore, sustained PI3-Kinase signalling in myeloid cells especially, elevates the osteoclastogenic potential of myeloid cells, leading to enhanced inflammatory local bone destruction. Therefore, although our study allows no direct translational conclusion since we used a conditional knockout approach, the therapeutic targeting of the PI3-Kinase pathway may be of benefit in preventing structural joint damage.

\section{INTRODUCTION}

Local and systemic bone loss is a hallmark of diseases such as osteoporosis, rheumatoid arthritis or spondylarthritis and often leads to disability and severely reduced quality of life and is therefore a serious health burden in humans. ${ }^{1-3}$ Such bone loss is almost exclusively mediated by a specialised type of cell, the osteoclast (OC). ${ }^{45}$ OCs are of haematopoietic origin and are derived from monocyte precursor cells. ${ }^{6}$ Many factors regulating differentiation and function of OCs have been described. ${ }^{6} 7$ Among them, the receptor activator of nuclear factor $\kappa-\mathrm{B}$ ligand (RANKL)-RANK pathway, as well as the macrophage colony-stimulating factor (M-CSF)-MCSF-receptor system, have been found to be essential for the generation of OCs under in vitro and in vivo conditions. ${ }^{8-11}$ A number of osteoclastogenic signalling pathways are integrated by the transcription factor nuclear factor of activated T-cells, cytoplasmic 1 (NFATc1), which regulates many OC-specific genes. ${ }^{12}{ }^{13}$ Nonetheless, the generation and function of OCs is not fully understood and several factors that influence these processes are yet to be elaborated.

Phosphoinositide-3-kinases (PI3Ks) are heterodimeric complexes, comprising a regulatory subunit and a p110 catalytic subunit that phosphorylate the membrane phospholipid phosphatidylinositol (PI). ${ }^{14}$ The resulting activation of protein kinase $\mathrm{B}$ (Akt) as well as of glycogen synthase kinase $3 \beta$ (GSK3 $\beta$ ) and the subsequent activation of genes regulated by these kinases mediate a variety of mechanisms such as inflammation, cell survival, cell migration, proliferation and cytoskeletal remodelling. ${ }^{14-16}$

Phosphatase and tensin homolog (PTEN) is a $53 \mathrm{kD}$ phospholipid phosphatase, whose main substrate is $\mathrm{PI}(3,4,5) \mathrm{P}_{3}$, the principal second messenger of the PI3K pathway. Therefore, PTEN is generally regarded as an antagonist of PI3Ks. PTEN is a potent tumour suppressor, ${ }^{17} 18$ and recent findings support the notion that the PI3K/PTEN pathway is intricately involved in the modulation of innate immune responses. ${ }^{19-21}$ Additionally, PTEN seems to be responsible for chemokine-dependent directed migration of various cell types, and also plays a major role in angiogenesis. ${ }^{22}{ }^{23} \mathrm{PI} 3 \mathrm{~K}$ has been shown to play a role in osteoclastogenesis, as demonstrated by the fact that $\mathrm{SH} 2$ domaincontaining inositol 5'-phosphatase (SHIP) deficient mice are osteoporotic due to increased generation of OCs. ${ }^{24}$ Additionally, inhibition of PI3K 
reduces osteoclastogenesis in vitro. ${ }^{26-28}$ PTEN has also been implicated in osteoclastogenesis, since transfection of RAW264.7 macrophages with a dominant negative version of PTEN increased, whereas overexpression of functional PTEN reduced osteoclastogenesis. ${ }^{29}$ Selective lack of PTEN in osteoblasts leads to enhanced bone formation as a result of prolonged survival and increased function of osteoblasts. ${ }^{30}$ However, the effect of PTEN deficiency in OC precursors or OCs in vivo, has not been investigated yet. We therefore analysed the effect of PTEN deficiency on osteoclastogenesis under homeostatic and inflammatory conditions by employing a conditional knockout mouse that lacks PTEN expression selectively in myeloid cells, subsequently referred to as myeloid-specific PTEN $^{-/-}$mice.

\section{MATERIALS AND METHODS \\ Animals}

Animals were identified by PCR from tail DNA using the following primers: human tumour necrosis factor (hTNF) transgene construct: 5'-TACCCCCTCCTTCAGACACC-3' and 5'-GCCCTTCA TAATATCCCCCA- $3^{\prime}$; Clinical signs of arthritis and body weight were determined once weekly. Animals were killed by cervical dislocation 12 weeks after birth. All animal procedures were approved by the local ethical committee. PTEN ${ }^{\text {flox/flox }}$ mice were provided by Dr Tak W Mak (University Health Network, Toronto, Canada). To selectively reduce PTEN expression in myeloid cells, PTEN ${ }^{\text {flox/flox }}$ mice were crossed with mice expressing the Cre recombinase under the control of the Lysozyme M (LysM) promoter (provided by Dr R Johnson, University of California San Diego, La Jolla, California, USA) to generate LysMCrePTEN ${ }^{\text {flox/flox }}$ (myeloid pten ${ }^{-1-}$ ) mice. LysMCrePTEN $^{\text {flox/flox }}$ and PTEN ${ }^{\text {flox/flox }}$ mice were backcrossed at least eight generations onto the $\mathrm{C} 57 \mathrm{BL} / 6 \mathrm{~J}$ background. These mice were crossed into Tg197 hTNF transgenic mice (hTNFtg; genetic background C57BL6 ${ }^{31}$ ) to obtain LysMCrePTEN ${ }^{\text {flox/flox }} / \mathrm{hTNFtg}$ mice. All data were generated from littermates.

\section{Clinical assessment of arthritis}

Clinical signs of arthritis, including grip strength and paw swelling were assessed weekly in mice starting 4 weeks after birth. Paw swelling was assessed by using a well-established semiquantitative score: $0=$ no swelling, $1=$ mild swelling of the toes and ankle, $2=$ moderate swelling of the toes and ankle, and $3=$ severe swelling of the toes and ankle. Grip strength of each paw was analysed on a wire mesh (3-mm in diameter) using a semiquantitative score from 0 to $-3(0=$ normal grip strength, $-1=$ mildly reduced grip strength, $-2=$ moderately reduced grip strength, $-3=$ severely reduced grip strength).

\section{Immunoblotting}

Proteins were separated by sodium dodecyl sulfate polyacrylamide gel electrophoresis (SDS-PAGE) and transferred to Immobilon-P membrane (Millipore, Billerica, Massachusetts, USA). The phosphorylation of AKT and GSK3 $\beta$ and the expression of PTEN and AKT C (Cell Signalling Technology, Danvers, Massachusetts, USA) was determined by overnight incubation at $4^{\circ}$. NFATc1 antibody (Santa Cruz Biotechnology) actin antibody (Sigma). Secondary antirabbit IgG-horseradish peroxidase (HRP)-conjugated antibody (Amersham Biosciences, Piscataway, New Jersey, USA). Membranes were washed and incubated with Supersignal West Femto substrate (Pierce Biotechnology, Rockford, Illinois, USA), solution and bands were detected by Fluor Chem HD2 (Alpha Innotec).

\section{Histological sections and histochemistry}

Hind paws were fixed and stained as previously described. ${ }^{32}$ Quantification of the areas of inflammation, H\&E sections were evaluated using an Axioskop 2 microscope (Carl Zeiss MicroImaging) and Osteomeasure Analysis System (OsteoMetrics). Tissue sections were stained with rat monoclonal antimacrophage (F4/80) antibody (Ab) (Serotec, Oxford, UK); diluted $1: 300$ ), followed by a biotinylated rabbit antirat IgG secondary Ab (Vector, Burlingame, California, USA). Proportions of $\mathrm{F} 4 / 80^{+} \mathrm{OC}$ precursors in hind paws as well as expression levels of NFATc1 in OCs were analysed using the TissueQuest software (Tissuegnostics, Vienna)

\section{Bone histomorphometry}

Histomorphometry was performed on methacrylate-embedded un-decalcified plastic sections after von Kossa and Goldner staining. ${ }^{33}$ Quantifications were performed by digital image analysis (OsteoMeasure).

\section{Dynamic labelling of bone}

At 16 weeks of age, mice were given two injections of calcein green (Sigma-Aldrich) $(30 \mathrm{mg} / \mathrm{kg}) 5$ days apart. Left tibial bones were embedded in methoxymethylmetacrylate. Measurements were performed on the entire marrow region within the cortical shell using OsteoMeasure, and the mineral apposition rate (MAR) $(\mu \mathrm{m} /$ day) was calculated.

\section{Statistical analysis}

Data are given as mean \pm SEM. Group mean values were compared by using the unpaired two-tailed Student $t$ test.

\section{Ex vivo osteoclastogenesis}

Bone marrow cells (BMC) were isolated and cultured for 3 days in $100 \mathrm{ng} / \mathrm{mL} \mathrm{M}$-CSF to enrich for monocytes/macrophages, and were then cultured in 10\% fetal calf serum/Dulbecco's modified Eagle's medium (FCS/DMEM) supplemented with $30 \mathrm{ng} / \mathrm{mL} \mathrm{M}-\mathrm{CSF}$ and $50 \mathrm{ng} / \mathrm{mL}$ RANKL (both from R\&D Systems McKinley Place NE, Minneapolis) for another 3-4 days. OCs were defined and detected as tartrate-resistant acid phosphatase (TRAP) ${ }^{+}$multinucleated cells ( $\geq 3$ nuclei). Wortmannin was added to the culture at a concentration of $1 \mu \mathrm{M}$. Bone resorption was carried out on $0.4 \mathrm{~mm}$-thick bovine cortical slices. The area of resorption per OC was calculated by dividing the total area of resorption by the total number of OCs using the Osteomeasure software.

\section{qPCR}

Total RNA was isolated from cultivated OCs using the RNeasy Mini kit (QIAGEN). $1 \mu \mathrm{g}$ total RNA was used for first strand cDNA synthesis (Amersham Biosciences) and $1 \mu \mathrm{L}$ cDNA was then used for PCR using the following primers: NFatc1: 5'-GACAGACATCGGGAGGAAGA- $3^{\prime}$ and $5^{\prime}$-AGCCTTCTCCAC GAAAATGA-3', cathepsin K: 5'-GGAAGAAGACTCACCAGAA GC- $3^{\prime}$ and $5^{\prime}$-GTCATATAGCCGCCTCCACAG- $3^{\prime}$; matrix metalloproteinase (MMP)-9: 5'-CCTGTGTGTTCCCGTTCATCT-3' and $5^{\prime}$-CGCTGGAATGATCTAAGCCCA-3'; TRAP: 5'-ACAGCC CCCACTCCCACCCT- $3^{\prime}$ and $5^{\prime}$-TCAGGGTCTGGGTCTCCTT GG-3'; calcitonin receptor: 5'-CATTCCTGTACTTGGTTGGC-3' and $5^{\prime}$-AGCAATCGACAAGGAGTGAC- ${ }^{\prime}$; and $\beta$-actin: $5^{\prime}$-TGTG ATGGTGGGAATGGGTCAG-3' and $5^{\prime}$-TTTGATGTCACGCAC GATTTCC-3'. Quantitative RT-PCR was performed using SYBR Green I and its detection by LightCycler (Roche Molecular Biochemicals). 
RESULTS

PTEN $^{-1-}$ bone marrow cells show increased osteoclastogenic capacity in vitro

To confirm the deletion efficiency of the conditional PTEN gene ablation we first analysed PTEN in OCs by immunoblotting. Therefore, we stimulated BMCs derived from PTEN ${ }^{\mathrm{fl} / \mathrm{fl}}$ LysM cre mice (myeloid $\mathrm{PTEN}^{-/}$) and littermate control wt mice with M-CSF and RANKL to generate OCs. Indeed, quantification revealed that deletion efficiency was more than 90\% (figure 1A). Moreover, we analysed effects on the deletion on PI3K signalling in myeloid PTEN ${ }^{-/-}$OCs. As expected, we found the phosphorylation of downstream targets of PI3K such as AKT or GSK3 $\beta$ to be constitutively enhanced in OCs derived from myeloid $\mathrm{PTEN}^{-/}$as compared with wt littermate controls (figure 1B).

We next investigated the osteoclastogenic potential of BMCs by evaluating the numbers of multinucleated $\mathrm{TRAP}^{+}$OCs and found a marked increase in OC numbers in cultures of BMCs derived from myeloid $\mathrm{PTEN}^{-/-}$compared with wt BMCs (figure 2A,B). Since the PI3K pathway is known to regulate proliferation and survival of cells, ${ }^{16}$ we investigated whether enhanced osteoclastogenesis was due to enhanced proliferation

A PTEN

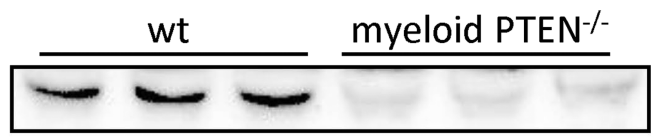

Actin
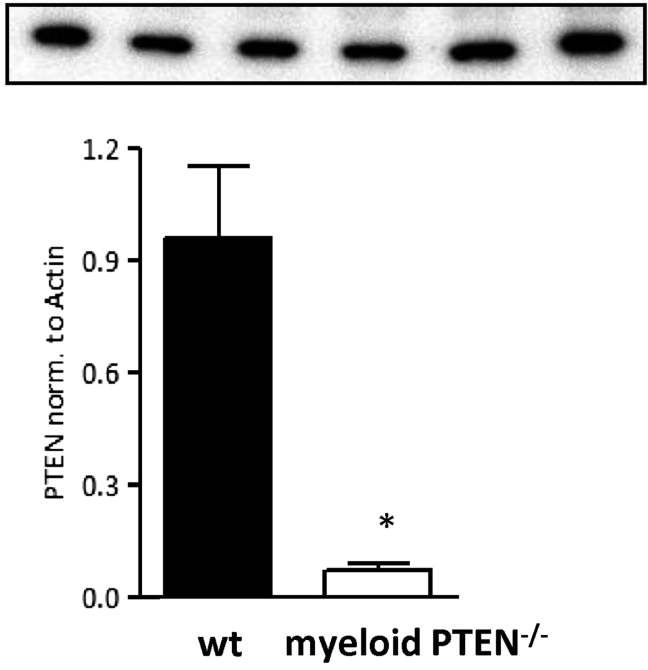

B

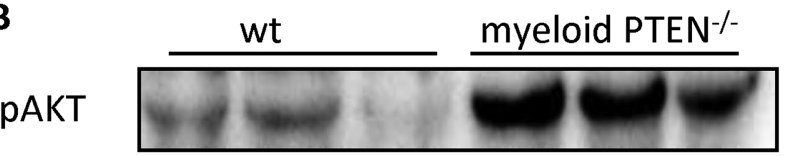
pGSK3ß

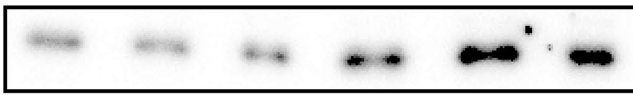

AKT

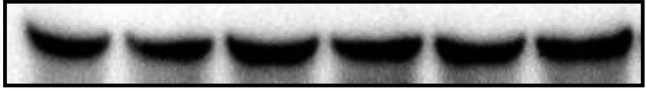

Figure 1 Osteoclasts (OCs) from myeloid phosphatase and tensin homolog $\left(\right.$ PTEN) ${ }^{-l-}$ bone marrow cells show efficient deletion of PTEN. (A) Analysis of the PTEN deletion efficiency in conditional PTEN knockout OCs; wildtype and myeloid PTEN ${ }^{-l-}$ OCs were tested for PTEN by immunoblotting. PTEN levels were quantified and normalised to actin. (B) Analysis of the phosphoinositide-3-kinase (PI3K) signalling axis in myeloid PTEN ${ }^{-1-}$ OCs; Activation of protein kinase B (AKT) and glycogen synthase kinase 3 beta (GSK3 $\beta$ ) was determined using phospho-specific antibodies. AKT was used as loading control. Plots are mean values $\pm S D$ of three independent experiments $\left({ }^{*} p \leq 0.05\right)$. of osteoclast precursors (pOCs) after stimulation with M-CSF. However, we did not detect any differences between the numbers of pOCs under these conditions in the two groups (fgure 2C). Additionally, when we labelled BMCs with carboxyfluorescein succinimidyl ester (CFSE) and measured CFSE dilution 3 days after stimulation with M-CSF, there was no difference between BMCs derived from myeloid $\mathrm{PTEN}^{-/}$as compared to wt (not shown).

In line with these findings, analysis of apoptosis by AnnexinV/7aminoactinomycin $\mathrm{D}(7 \mathrm{AAD})$-staining did not reveal differences in the amount of apoptotic pOCs from myeloid $\mathrm{PTEN}^{-/}$as compared with wt (figure 2D). However, activation of PI3-kinase after stimulation with M-CSF is important for survival in wt as well as in myeloid $\mathrm{PTEN}^{-/}$, as inhibition of this pathway with wortmannin led to increased proportions of AnnexinV/7AAD positive cells in both genotypes (see online supplementary figure S1). Taken together, these data indicate that the absence of PTEN has no effect on pOC proliferation or apoptosis.

We subsequently analysed the role of PTEN on survival of OCs. We found that osteoclastogenesis in BMCs from myeloid $\mathrm{PTEN}^{--}$and wt mice peaked 3 days after stimulation with RANKL with the number of OCs declining after this time point in similar fashion in both groups (figure 2E). However, osteoclastogenesis started earlier and the number of resulting OCs was higher when we used myeloid $\mathrm{PTEN}^{-/}$BMCs to differentiate OCs. This suggests that enhanced osteoclastogenesis was due to enhanced RANKL-mediated differentiation of OCs and not due to enhanced generation of pOCs or enhanced survival of differentiated OCs.

To analyse the molecular basis for enhanced RANKL-mediated differentiation of pOCs to OCs in myeloid PTEN ${ }^{-/}$BMCs, we measured NFATc1 and indeed, detected a marked upregulation of NFATc1 in myeloid PTEN ${ }^{-/}$BMCs compared with wt by qPCR as well as western blotting (figure $2 \mathrm{~F}, \mathrm{G}$ ). We next analysed mRNA expression of other OC-related genes and did not find significant differences in the expression of calcitonin-receptor or the M-CSF-receptor macrophage colony-stimulating factor receptor/CD115 (cFMS) between myeloid $\mathrm{PTEN}^{-/}$and wt BMCs. However, mRNA of several OC effector genes including MMP9, TRAP and cathepsin K, was significantly overexpressed in OCs derived from myeloid $\mathrm{PTEN}^{-/}$cells compared with wt cells (figure 3A-E).

We also tested the capacity of myeloid PTEN $^{-/}$OCs to resorb bone. We found no difference between the two groups (figure 3F). Taken together, we found that PTEN is important in regulating osteoclastogenesis, but has no effect on OC function per se.

\section{Increased in vivo osteoclastogenesis in myeloid-specific PTEN $^{-1-}$ mice}

Next, we asked whether PTEN deficiency also affects in vivo osteoclastogenesis. To answer this question, we analysed histological sections of tibial bones from 16-week-old myeloidspecific $\mathrm{PTEN}^{-/-}$mice and wt mice. We found a significant increase in the number of OCs per bone perimeter $(\mathrm{NOc} / \mathrm{BPm})$ as well as OC surface per bone surface (OcS/BS) in myeloid-PTEN ${ }^{-/-}$mice compared with wt animals (figure 4A). In line with our in vitro data, this indicates that the absence of PTEN also enhances osteoclastogenesis in vivo.

PTEN deficiency in osteoblasts has been reported to increase bone formation via enhanced proliferation and function of osteoblasts. ${ }^{30}$ Therefore, we asked whether the absence of PTEN restricted only to myeloid cells, but not affecting mesenchymal osteoblasts has indirect effects on osteoblast function in vivo. Indeed, when we analysed the MAR, we detected a 
A
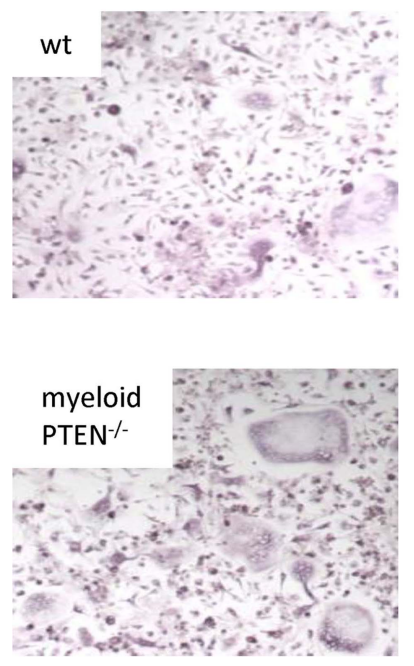

B

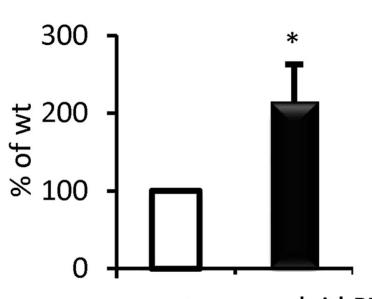

c

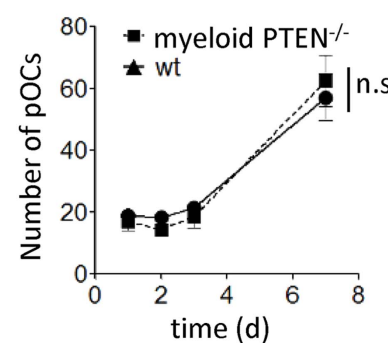

D

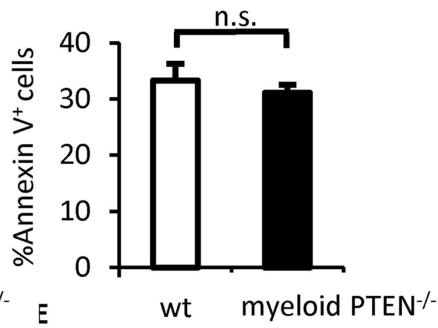

$100]=$ myeloid PTEN $-1-$

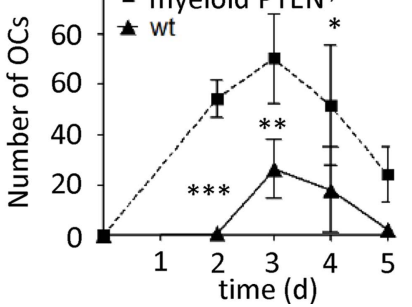

$\mathbf{F}$
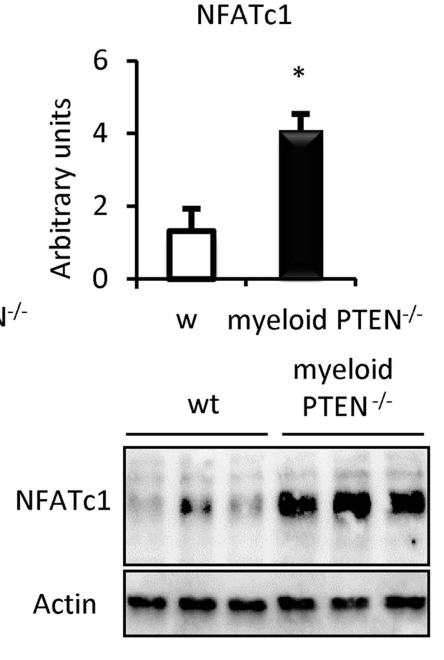

Figure 2 Myeloid phosphatase and tensin homolog (PTEN) ${ }^{-l-}$ bone marrow cells show an increased osteoclastogenic capacity in vitro. (A and B) Ex vivo osteoclastogenesis using BMCs of wildtype (wt) and myeloid PTEN ${ }^{-1}$ mice 4 days after the addition of receptor activator of nuclear factor $\kappa-B$ ligand (RANKL); (A) representative tartrate-resistant acid phosphatase (TRAP) stainings of wt and myeloid PTEN ${ }^{-1-}$ osteoclasts (OCs) (original magnification 100x). (B) Quantitative analysis of TRAP ${ }^{+}$multinucleated cells. (C) Number of osteoclast precursors (pOCS) of wt and myeloid PTEN ${ }^{-1-}$ BMCs after stimulation with macrophage colony-stimulating factor (M-CSF). Plots show mean values \pm SD of three mice per genotype.

(D) Flow cytometric analysis of Annexin ${ }^{+}$BMCs after stimulation with M-CSF for 3 days. Plots are mean values \pm SD of three mice per genotype.

(E) Analysis of $O C$ numbers over time after addition of RANKL to the culture. (error bars $\pm S E M ;{ }^{*} p \leq 0.05 ;{ }^{* *} p \leq 0,01 ;{ }^{* * *} p \leq 0.001$ ). (F and G) analysis of nuclear factor of activated T-cells, cytoplasmic 1 (NFATC1) expression in wt and PTEN ${ }^{-1-}$ BMCs after the addition of RANKL by qPCR $(\mathrm{F})$ and western blot $(\mathrm{G})$. Plot is mean values $\pm \mathrm{SD}(\mathrm{n}=3)$.

significant increase in myeloid $\mathrm{PTEN}^{-/-}$mice compared with wt mice (figure 4B).

We could not detect any differences between myeloid PTEN ${ }^{-/-}$ mice and wt animals in bone volume per tissue volume (BV/TV), trabecular thickness (TbTh), trabecular number $(\mathrm{TbN})$ or trabecular separation $(\mathrm{TbSp})$, demonstrating an unchanged net balance of bone turnover (figure 4C).

\section{Myeloid PTEN ${ }^{-I-}$ mice suffer from severely reduced grip strength despite similar paw swelling, as compared with wt mice}

We next evaluated whether there is an impact of PTEN deficiency in myeloid cells on arthritis development, especially with regard to local osteoclastogenesis and bone destruction. Therefore, we crossed hTNF transgenic (hTNFtg) mice, which
Figure 3 Receptor activator of nuclear factor $\kappa$-B ligand -stimulated myeloid Phosphatase and Tensin homolog (PTEN) $)^{-l-}$ bone marrow cells display enhanced expression of osteoclast (OC)-related genes. (A-E) qPCR analysis of the indicated mRNAs. Plots show mean values \pm SD, and are representative of at least 3 independent experiments. (F) Pit resorption assay of OCs derived from wildtype and myeloid PTEN ${ }^{-1-}$ mice. The total area of resorption was divided by the total number of $\mathrm{OC}$, resulting in area of resorption per $\mathrm{OC}$, using the Osteomeasure software. CathK, cathepsin K; cFMS, macrophage colony-stimulating factor receptor/ CD115; MMP9, matrix metalloproteinase 9; TRAP, tartrateresistant acid phosphatase.

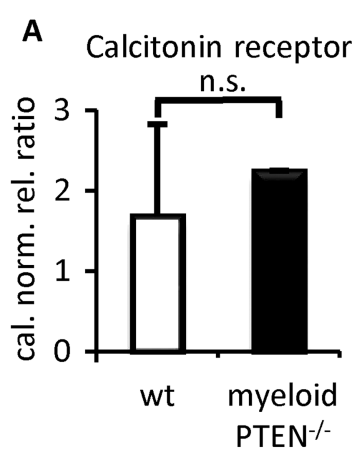

B

CFMS

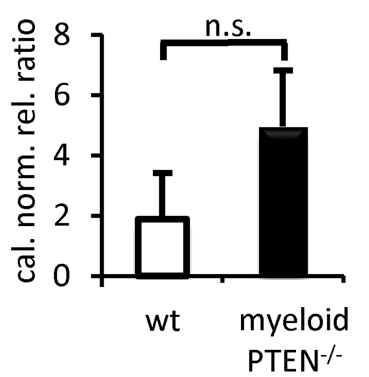

E

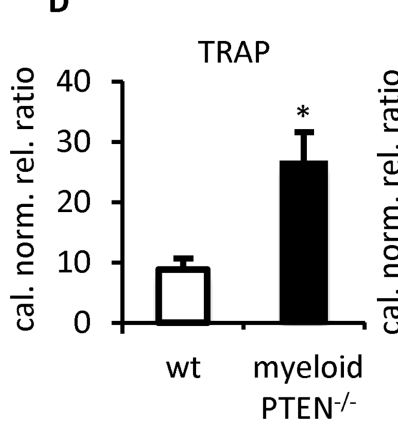

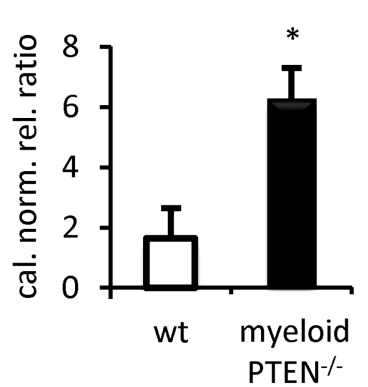

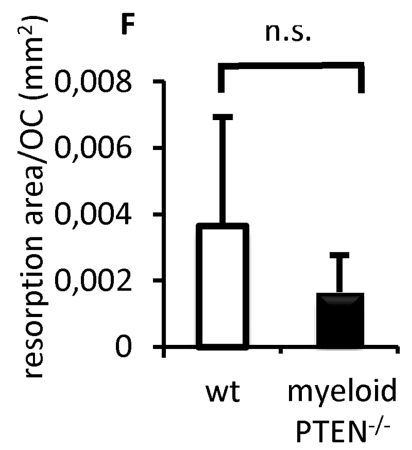



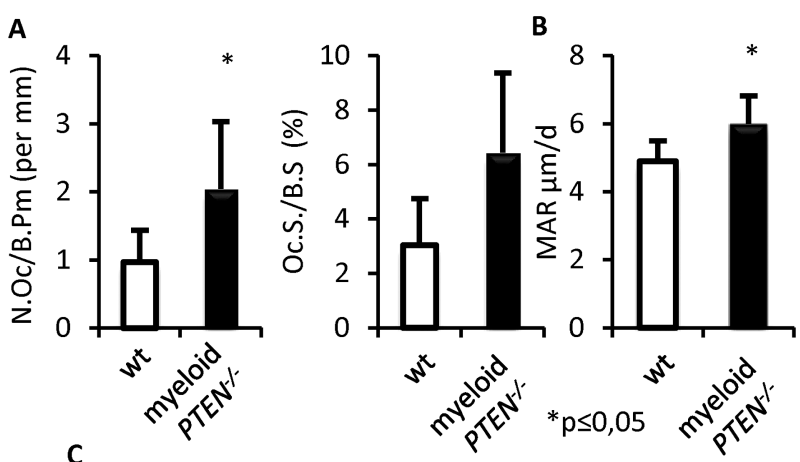

C
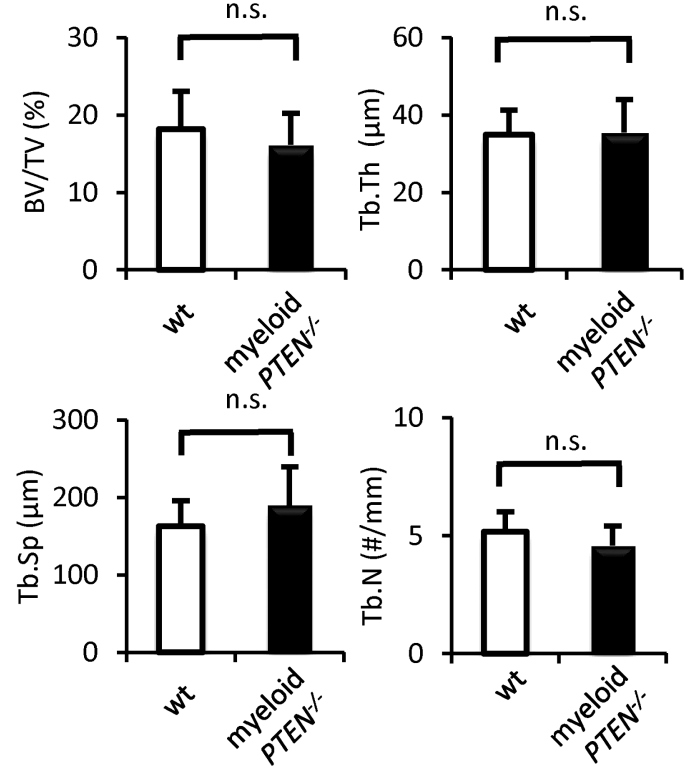

Figure 4 Increased osteoclastogenesis in vivo in myeloid phosphatase and tensin homolog (PTEN) ${ }^{-/-}$mice. (A) Bone histomorphometry of osteoclast-related bone parameters of wildtype (wt) and myeloid $\mathrm{PTEN}^{-1-}$ mice (age: 16 weeks). Plots are mean values \pm SD $(n=7)$ $\left({ }^{*} p \leq 0.05\right)$. (B) Quantitative expression of the mineral apposition rate in wt and myeloid PTEN ${ }^{-1-}$ mice (age: 16 weeks) $\left({ }^{*} p \leq 0.05\right)$. (C) Bone histomorphometry of wt and myeloid PTEN ${ }^{-1-}$ mice (age: 16 weeks). Plots are mean values $\pm S D(n=7)\left({ }^{*} p \leq 0.05\right)$.
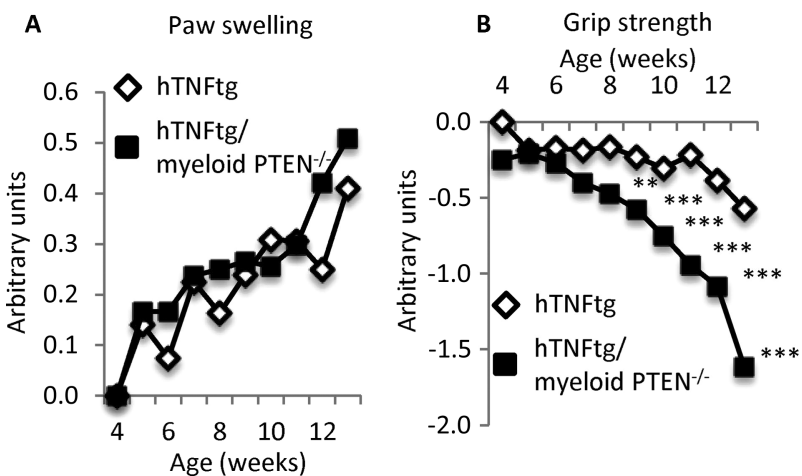

Figure 5 Human tumour necrosis factor-transgenic (hTNFtg)/myeloid phosphatase and tensin homolog (PTEN) ${ }^{-1-}$ mice display severely reduced grip strength despite similar paw swelling compared with wildtype mice. (A and B) Clinical assessment of paw swelling (A) and grip strength (B) in hTNFtg $(n=15)$ and hTNFtg/myeloid PTEN ${ }^{-l-}$ mice $(n=17)$ over time. Plots show mean values $\pm S D\left({ }^{*} p \leq 0.05 ;{ }^{* *} p \leq 0.01\right.$; $\left.{ }^{* * *} \mathrm{p} \leq 0.001\right)$. develop a symmetrical, erosive arthritis as a consequence of constitutive overexpression of the hTNF gene, ${ }^{31}$ with myeloid $\mathrm{PTEN}^{-/-}$mice generating hTNFtg/myeloid PTEN ${ }^{-/}$animals. When we analysed clinical signs of arthritis over time, we did not detect significant differences in the extent of paw swelling between hTNFtg and hTNFtg/myeloid $\mathrm{PTEN}^{-/}$littermate animals (figure 5A). By contrast, there was a highly significant difference in grip strength starting from week 8, with grip strength deteriorating earlier and more severely in hTNFtg/ myeloid PTEN ${ }^{-/-}$mice than in hTNFtg animals (figure 5B).

\section{Increased joint destruction in myeloid PTEN $^{-1-}$}

To determine the impact of PTEN on TNF-induced arthritis in more detail, we analysed the histological sections of hind paws. We found a numerical but not significant increase of synovial inflammation in hTNFtg/myeloid PTEN ${ }^{-/}$when compared to hTNFtg mice (figure 6A).

Since we have shown that PTEN effects NFATc1 expression in OCs in vitro, we next analysed the expression of NFATc1 in OCs under inflammatory conditions in vivo. Indeed, we found increased NFATc1 protein levels in OCs in the inflamed synovial membrane of hTNFtg/myeloid $\mathrm{PTEN}^{-/}$mice (figure 6B). Moreover, there was a dramatic increase in OC numbers in hTNFtg/myeloid PTEN $^{-/}$mice accompanied by a marked increase in local bone destruction (figure 6C,D).

We also investigated if pOC numbers in the inflamed synovial membrane differed between hTNFtg/myeloid $\mathrm{PTEN}^{-/-}$and hTNFtg mice. In line with our in vitro data, we did not find differences in relative numbers of pOC or $\mathrm{Gr}^{+}$granulocytes in the synovial membranes of the two groups of animals (figure 6E).

Interestingly, in hTNFtg/myeloid $\mathrm{PTEN}^{-/-}$mice, osteoclastogenesis was increased more profoundly than synovial inflammation, as we could identify significantly more OCs per area of inflammation than in hTNFtg animals (figure 6G). However, the relation between the size of erosions and number of OCs was not different between the groups (figure 6H), suggesting that the bone resorbing capacity of individual OCs from hTNFtg/myeloid PTEN ${ }^{-/-}$animals was not different compared with that of OCs from hTNFtg animals.

\section{DISCUSSION}

In this study, we provide evidence for an important role of the PI3K/PTEN pathway in OCs as deficiency of PTEN, and subsequent sustained PI3K activity in myeloid cells including pOCs leads to increased osteoclastogenesis in vitro and in vivo.

Our data show that absence of PTEN in myeloid cells augments the potential of monocytic OC precursors to differentiate into OCs under various conditions. Although proliferation and survival of myeloid PTEN ${ }^{-/-}$pOCS is not altered under in vitro conditions, their RANKL induced differentiation into OCs is highly increased. This is accompanied by an increased induction of NFATc1, known to be a master regulator of the RANKL-RANK pathway. ${ }^{13}$ Of note, neither the life span nor the resorptive capacity of myeloid $\mathrm{PTEN}^{-/-} \mathrm{OCs}$ is affected. This is also observed under homeostatic conditions in vivo, as OCs numbers are markedly increased in bones of myeloid $\mathrm{PTEN}^{-/-}$mice. However, these mice do not become osteopenic due to compensation by a hyperactivity of osteoblasts, as shown by the significant increase in bone apposition rates in myeloid PTEN $^{-/-}$mice.

The data we obtained in our experiments are different from those reported for another inhibitor of PI3K, SHIP. SHIP-deficient animals are osteoporotic due to increased 
Figure 6 Joint inflammation and joint destruction are dissociated in human TNF-transgenic (hTNFtg)/ myeloid phosphatase and tensin homolog (PTEN) ${ }^{-1-}$ mice.

(A) Quantitative histomorphometric analysis of inflammation in the synovial membrane of hTNFtg $(n=15)$ and hTNFtg/myeloid PTEN ${ }^{-/-}$mice $(n=11)$. (B) Mean intensity of nuclear factor of activated T-cells, cytoplasmic 1 (NFATc1) expression in osteoclasts (OCs) in the synovial membrane of hTNFtg and hTNFtg/myeloid PTEN ${ }^{-1-}$ mice. Mean area of erosion (C) and number of OCs (D) in the tarsal area of the hind paws of hTNFtg and hTNFtg/ myeloid PTEN ${ }^{-1-}$ mice ( $\left.{ }^{*} p \leq 0.05\right)$. (E) Analysis of the percentage of $\mathrm{F} 4 / 80^{+}$ cells shown as percent of total cells in the synovial membrane of hTNFtg and hTNFtg/myeloid PTEN ${ }^{-l-}$ mice. (F) Analysis of the percentage of $\mathrm{Gr}^{+}$ cells shown as percent of total cells in the synovial membrane of hTNFtg and hTNFtg/myeloid PTEN ${ }^{-I-}$ mice. (G) Analysis of the number of OCs per area of inflammation in the synovial membrane of hTNFtg and hTNFtg/ myeloid PTEN ${ }^{-1-}$ mice. ( ${ }^{*} p \leq 0.05$; $\left.{ }^{* *} p \leq 0.01 ;{ }^{* * *} p \leq 0.001\right)$. (H) Analysis of the area of erosion caused by OCS in the synovial membrane of hTNFtg and hTNFtg/myeloid PTEN ${ }^{-/-}$mice.
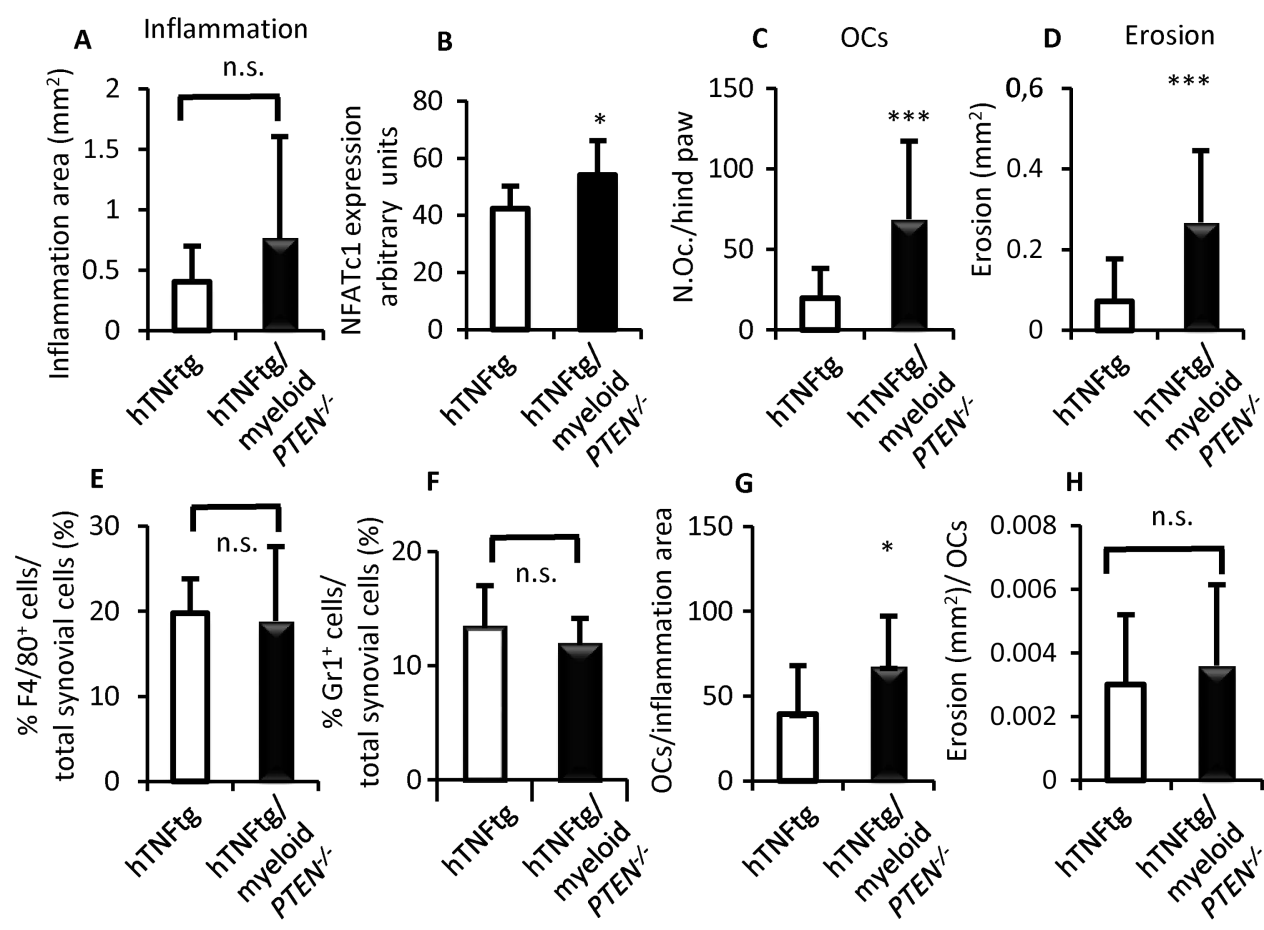

H
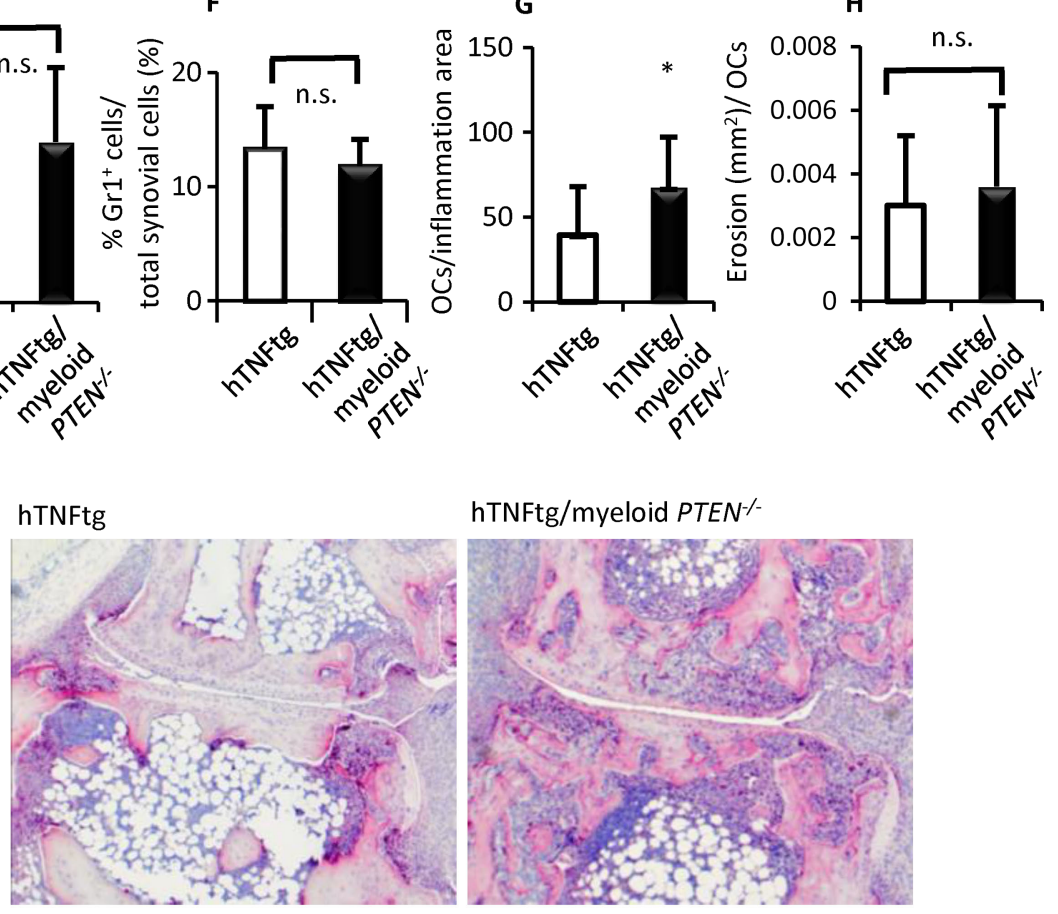

hTNFtg/myeloid PTEN-/-

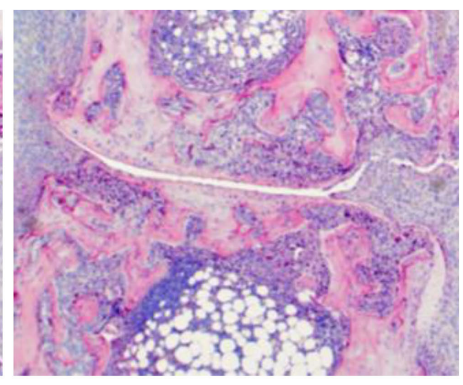

osteoclastogenesis and OC function. ${ }^{24}$ It should be noted, however, that the mice used in the above-mentioned study exhibited a constitutive and generalised SHIP deficiency, whereas in our setting, PTEN deficiency is conditional and selectively affects myeloid cells. Nevertheless, there seem to be specific differences in the signalling pathways that are differentially inhibited by SHIP and PTEN. Experiments using human macrophages have clearly shown that activation of PI3-kinase regulates the survival of macrophages. ${ }^{34}$ We could also confirm this finding, since the inhibition of PI3-kinase signalling by wortmannin increased the number of apoptotic cells in wt and myeloid PTEN ${ }^{-1-}$ BMCs. However, deletion of PTEN does not lead to reduced apoptosis in these cells, a phenomenon that has also been noted in SHIP-deficient BMCs. ${ }^{25}$ Additionally, blood counts of monocytes and neutrophils, as well as the relative number of CD11b cells in the spleen were not different between wt and myeloid PTEN ${ }^{-/-}$mice (see online supplementary figure S2), arguing against a major proliferative or survival advantage of myeloid cells in myeloid PTEN $^{-/}$compared to wt. Potential effects of SHIP deficiency involving the mesenchymal cell compartment, which plays a role in bone biology, namely osteoblasts and osteocytes, could at least partly contribute to the reduced bone mass detected in these mice.

Interestingly, the importance of PTEN in terminal differentiation of OCs under inflammatory conditions can also be observed in the bones comprising the involved joints of myeloid
$\mathrm{PTEN}^{-}$animals overexpressing hTNF. Although synovial inflammation is only insignificantly increased, hTNFtg/myeloid $\mathrm{PTEN}^{-/-}$mice suffer from considerably increased local bone destruction. This is associated with highly decreased grip strength scores of hTNFtg/myeloid PTEN $^{-/-}$mice compared with hTNFtg animals. In line with the data obtained previously, the numbers of pOCs in the inflamed synovial membrane are not different from those in hTNFtg animals. The numbers of mature OCs, however, are markedly increased in hTNFtg/ myeloid PTEN $^{-/}$mice. This argues for a specific role of the PI3K/PTEN axis in RANKL/RANK-driven osteoclastogenesis by facilitating the generation of bone resorbing OCs from the OC precursor pool (see online supplementary figure S3). Given the importance of bone erosion for the loss of function in patients with RA we think that the discovery of mechanisms leading to bone erosions independent from inflammation is of great importance for the development of new therapeutic strategies. Although it is difficult to draw a direct translational conclusion based on these results alone, they nonetheless suggest that restricting PI3K activity in myeloid cells might be useful in inhibiting local bone destruction in arthritis.

Correction notice This article has been corrected since it was published Online First. The funding section has been updated.

Acknowledgements We thank Tetyana Shvets, Aurica Jelinek for expert technical assistance. We thank Dr George Kollias for providing the hTNFtg mice. 
Contributors $S B, G S, J S S$ and $K R$ : designed the experiments. $S B, M F, A P, T L, E S$, $V S, J B, P M, B N$ and GS: performed experiments. SB, AP and GS: analysed results. SB and GS: made the figures. SB, GS, JSS and KR wrote the paper.

Funding This research has received support from the Innovative Medicines Initiative Joint Undertaking under grant agreement number 115142 (BTCure), resources of which are composed of financial contribution from the European Union's Seventh Framework Programme and EFPIA companies' in kind contribution, by the Coordination Theme 1 (Health) of the European Community's FP7; Grant Agreement number HEALTH-F2-2008-223404 (Masterswitch), and by a grant from the Austrian science fund (FWF), number P 23730.

Competing interests None.

Provenance and peer review Not commissioned; externally peer reviewed.

\section{REFERENCES}

1 Scott DL, Pugner K, Kaarela K, et al. The links between joint damage and disability in rheumatoid arthritis. Rheumatology (Oxford) 2000;39:122-32.

2 Anandarajah AP, Schwarz EM. Bone loss in the spondyloarthropathies: role of osteoclast, RANKL, RANK and OPG in the spondyloarthropathies. Adv Exp Med Biol 2009;649:85-99

3 Sanchez-Riera L, Wilson N, Kamalaraj N, et al. Osteoporosis and fragility fractures. Best Pract Res Clin Rheumatol 2011;24:793-810.

4 Kong YY, Feige U, Sarosi I, et al. Activated T cells regulate bone loss and joint destruction in adjuvant arthritis through osteoprotegerin ligand. Nature 1999;402:304-9.

5 Redlich K, Hayer S, Ricci R, et al. Osteoclasts are essential for TNF-alpha-mediated joint destruction. J Clin Invest 2002;110:1419-27.

6 Boyle WJ, Simonet WS, Lacey DL. Osteoclast differentiation and activation. Nature 2003:423:337-42.

7 Teitelbaum SL, Ross FP. Genetic regulation of osteoclast development and function. Nat Rev Genet 2003;4:638-49.

8 Kong YY, Yoshida H, Sarosi I, et al. OPGL is a key regulator of osteoclastogenesis, lymphocyte development and lymph-node organogenesis. Nature 1999:397:315-23.

9 Simonet WS, Lacey DL, Dunstan CR, et al. Osteoprotegerin: a novel secreted protein involved in the regulation of bone density. Cell 1997;89:309-19.

10 Yasuda $\mathrm{H}$, Shima N, Nakagawa $\mathrm{N}$, et al. Osteoclast differentiation factor is a ligand for osteoprotegerin/osteoclastogenesis-inhibitory factor and is identical to TRANCE/ RANKL. Proc Natl Acad Sci USA 1998;95:3597-602.

11 Wiktor-Jedrzejczak W, Bartocci A, Ferrante AW Jr, et al. Total absence of colony-stimulating factor 1 in the macrophage-deficient osteopetrotic (op/op) mouse. Proc Natl Acad Sci USA 1990;87:4828-32.

12 Takayanagi H, Kim S, Koga T, et al. Induction and activation of the transcription factor NFATC1 (NFAT2) integrate RANKL signaling in terminal differentiation of osteoclasts. Dev Cell 2002;3:889-901.

13 Negishi-Koga T, Takayanagi H. Ca2+-NFATc1 signaling is an essential axis of osteoclast differentiation. Immunol Rev 2009;231:241-56.

14 Vogt PK, Hart JR, Gymnopoulos M, et al. Phosphatidylinositol 3-kinase: the oncoprotein. Curr Top Microbiol Immunol 2010;347:79-104.
15 Okkenhaug K, Vanhaesebroeck B. PI3K in lymphocyte development, differentiation and activation. Nat Rev Immunol 2003;3:317-30.

16 Hawkins PT, Anderson KE, Davidson K, et al. Signalling through Class I PI3Ks in mammalian cells. Biochem Soc Trans 2006;34(Pt 5):647-62.

17 Leslie NR, Downes CP. PTEN function: how normal cells control it and tumour cells lose it. Biochem J 2004;382(Pt 1):1-11.

18 Parsons R. Human cancer, PTEN and the PI-3 kinase pathway. Semin Cell Dev Biol 2004:15:171-6.

19 Schabbauer G, Luyendyk J, Crozat K, et al. TLR4/CD14-mediated PI3K activation is an essential component of interferon-dependent VSV resistance in macrophages. Mol Immunol 2008;45:2790-6.

20 Gunzl P, Schabbauer G. Recent advances in the genetic analysis of PTEN and PI3K innate immune properties. Immunobiology 2008;213:759-65.

21 Gunzl P, Bauer K, Hainzl E, et al. Anti-inflammatory properties of the PI3K pathway are mediated by IL-10/DUSP regulation. J Leukoc Biol 2010;88:1259-69.

22 Heit B, Robbins SM, Downey CM, et al. PTEN functions to 'prioritize' chemotactic cues and prevent 'distraction' in migrating neutrophils. Nat Immunol 2008;9: $743-52$.

23 Subramanian KK, Jia Y, Zhu D, et al. Tumor suppressor PTEN is a physiologic suppressor of chemoattractant-mediated neutrophil functions. Blood 2007;109:4028-37.

24 Takeshita S, Namba N, Zhao JJ, et al. SHIP-deficient mice are severely osteoporotic due to increased numbers of hyper-resorptive osteoclasts. Nat Med 2002;8:943-9.

25 Zhou P, Kitaura H, Teitelbaum SL, et al. SHIP1 negatively regulates proliferation of osteoclast precursors via Akt-dependent alterations in D-type cyclins and p27. J Immunol 2006;177:8777-84.

26 Moon JB, Kim JH, Kim K, et al. Akt induces osteoclast differentiation through regulating the GSK3beta/NFATC1 signaling cascade. J Immunol 2012;188:163-9.

27 Cao H, Yu S, Yao Z, et al. Activating transcription factor 4 regulates osteoclast differentiation in mice. J Clin Invest 2010;120:2755-66.

28 Gingery A, Bradley E, Shaw A, et al. Phosphatidylinositol 3-kinase coordinately activates the MEK/ERK and AKT/NFkappaB pathways to maintain osteoclast survival. J Cell Biochem 2003;89:165-79.

29 Sugatani T, Alvarez U, Hruska KA. PTEN regulates RANKL- and osteopontin-stimulated signal transduction during osteoclast differentiation and cell motility. J Biol Chem 2003;278:5001-8.

30 Liu X, Bruxvoort KJ, Zylstra CR, et al. Lifelong accumulation of bone in mice lacking Pten in osteoblasts. Proc Natl Acad Sci USA 2007;104:2259-64.

31 Keffer J, Probert L, Cazlaris H, et al. Transgenic mice expressing human tumour necrosis factor: a predictive genetic model of arthritis. EMBO J 1991;10: 4025-31.

32 Bluml S, Binder NB, Niederreiter B, et al. Anti-inflammatory effects of TNF on hematopoietic cells in the development of erosive arthritis. Arthritis Rheum 2010;62:1608-19.

33 Binder NB, Niederreiter B, Hoffmann O, et al. Estrogen-dependent and C-C chemokine receptor-2-dependent pathways determine osteoclast behavio $r$ in osteoporosis. Nat Med 2009;15:417-24.

34 Liu H, Perlman H, Pagliari LJ, et al. Constitutively activated Akt-1 is vital for the survival of human monocyte-differentiated macrophages. Role of Mcl-1, independent of nuclear factor (NF)-kappaB, Bad, or caspase activation. J Exp Med 2001;194:113-26. 